\title{
T cell vaccines for microbial infections
}

\author{
Harriet L Robinson \& Rama Rao Amara
}

Vaccination, or the deliberate induction of protective immunity by administering nonpathogenic forms of a microbe or its antigens to induce a memory immune response, is the world's most cost-effective medical procedure for preventing morbidity and mortality caused by infectious disease ${ }^{1}$. Historically, most vaccines have worked by eliciting long-lived plasma cells. These cells produce antibodies that limit disease by neutralizing a toxin or blocking the spread of the infectious agent. For these 'B cell vaccines,' the immunological marker, or correlate, for protection is the titer of protective antibodies. With the discovery of HIV/AIDS, vaccine development has been confronted by an agent that is not easily blocked by antibody ${ }^{2}$. To overcome this, researchers who are developing HIV/AIDS vaccines have turned to the elicitation of cellular immunity, or 'T cell vaccines,' which recognize and kill infected cells ${ }^{3,4}$.

The success of $\mathrm{T}$ cell vaccines depends on two types of T cells: CD8 $\mathrm{T}$ cells and CD4 T cells. CD8 T cells limit infections by recognizing and killing infected cells and secreting antiviral cytokines, and are frequently referred to as cytotoxic $\mathrm{T}$ cells $\left(\mathrm{CTL}\right.$ or $\mathrm{T}_{\mathrm{C}}$ ). $\mathrm{CD} 4 \mathrm{~T}$ cells provide growth factors and signals for the generation ${ }^{5-7}$ and maintenance ${ }^{8-10}$ of CD8 $\mathrm{T}$ cells and are referred to as helper cells or $\mathrm{T}_{\mathrm{H}}$. The immunological foundations for $\mathrm{B}$ cell and $\mathrm{T}$ cell vaccines are not mutually exclusive and most antimicrobial vaccines prime both humoral and cellular immunity.

\section{Overview of $\mathrm{T}$ cell vaccines}

The forte of $\mathrm{T}$ cell vaccines is their ability to kill cells that are harboring a chronic infectious agent. Killing is affinity dependent and requires direct contact between a CD8 cell and its target for several minutes to an hour, after which the CD8 cell remains intact for additional killing, whereas the target cell undergoes apoptotic death. Activated T cells also produce cytokines and chemokines that can act in highly infection-specific mechanisms to interfere with the spread or replication of a microbe ${ }^{11-13}$.

$\mathrm{T}$ cells recognize peptides derived from foreign proteins that are processed and presented on the histocompatibility antigens of a vaccinated animal or human. CD8 $\mathrm{T}$ cells recognize peptides that are 8-11 amino acids long (epitopes) presented by major histocompatibility complex (MHC) class I antigens. Examples of MHC class I antigens are human leukocyte antigens (HLA) or their cognate in mice, $\mathrm{H}-2$ antigens. CD4 $\mathrm{T}$ cells recognize epitopes that are 10-18 amino acids long presented by MHC class II histocompatibility antigens (for example, DR, DP and DQ antigens of humans and I-A and I-E antigens of mice). Each member of a human or animal population recognizes only those epitopes that can be presented by his or her MHC type. In contrast to protective antibodies, which target exposed regions of external proteins, protective $\mathrm{CD} 8$ cells can target any region of any internal or external microbial

Emory Vaccine Center and Yerkes National Primate Research Center, Emory University, Atlanta, Georgia 30329, USA.

(e-mail: hrobins@rmy.emory.edu)

Published online 20 March 2005; doi:10/nm1212 protein that can undergo class I presentation. In our experience with HIV immunogens in vaccinated macaques, CD4 and CD8 epitopes are spread throughout proteins with about one CD4 epitope for every 100 amino acids of protein and one CD8 epitope for every 200-300 amino acids of protein ${ }^{14-17}$.

Because $T$ cell responses are dependent on histocompatibility antigens, which are different in different members of a population, $T$ cell vaccines can elicit highly variable immune responses, especially if the response is limited to a relatively short stretch of amino acids. Figure la shows data from macaques on the variability in vaccine-elicited $\mathrm{T}$ cells for the $\sim 500$ amino-acid HIV Gag protein compared with the variability seen for responses to the current smallpox vaccine, a vaccine that encodes about 200 proteins. At the peak vaccine response for Gag, the frequencies of responding CD8 $\mathrm{T}$ cells varied by $>100$-fold and the frequencies of responding CD4 cells by $\sim 20$-fold. In contrast, the CD8 and CD4 responses for the smallpox vaccine varied by $<10$-fold. Both the number of recognized epitopes and the immunodominance of the recognized epitopes contribute to this variability for CD8 responses. Strongly immunodominant epitopes elicit high frequencies of responding cells and limit responses against subdominant epitopes ${ }^{18}$. CD4 responses also show immunodominance, but this is much less pronounced than for CD8 $\mathrm{T}$ cells ${ }^{14}$. The high variability of $\mathrm{T}$ cell responses to Gag contrasts with the variability for antibody responses to Env (also a single HIV protein), which are remarkably uniform (Fig. 1a).

A major limitation of $\mathrm{T}$ cell vaccines is the ability of pathogens to undergo $\mathrm{T}$ cell escape by mutating a target epitope so that it is no longer recognized by a responding $\mathrm{CD} 8 \mathrm{~T}$ cell ${ }^{19}$. The most effective way to minimize microbial escape from a $\mathrm{T}$ cell response is to use vaccine inserts that are sufficiently large for each vaccinated animal or person to recognize multiple epitopes (we use inserts of $>2,000$ amino acids). A second limitation of $\mathrm{T}$ cell vaccines is the potential for $\mathrm{T}$ cells to become exhausted by high levels of persisting antigen ${ }^{20-22}$. Indeed, the hallmarks for successful $\mathrm{T}$ cell-mediated protection of macaques against an immunodeficiency virus are (i) stable, low-level CD8 memory responses that are only minimally evolving in response to a contained infection ${ }^{23}$ and (ii) $\mathrm{CD} 4$ and CD8 T cells that maintain their 'vigor', as evidenced by the ability to produce interleukin (IL)-2 (refs. 23-25) and perforin ${ }^{25}$ after restimulation. 
a

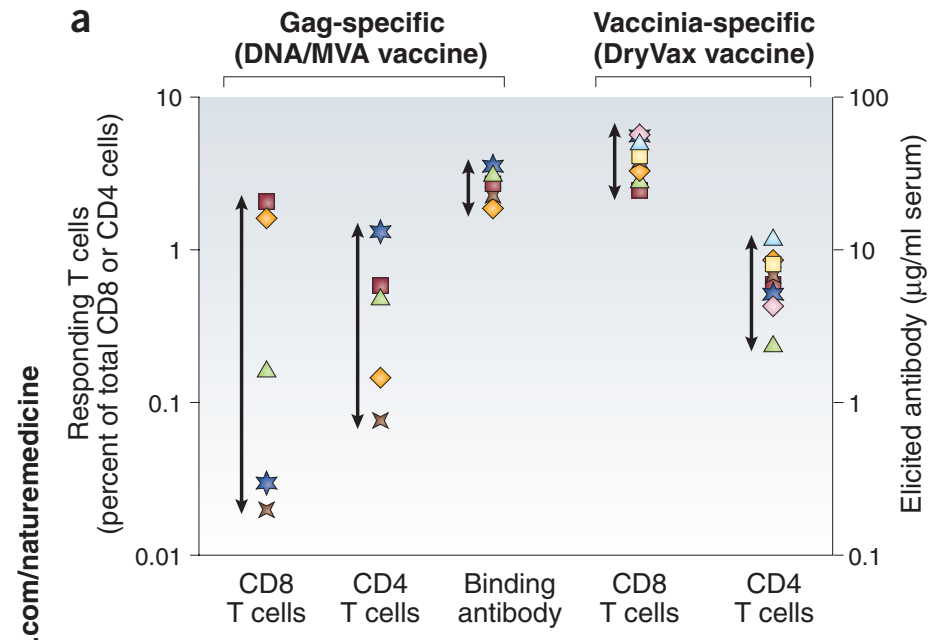

b

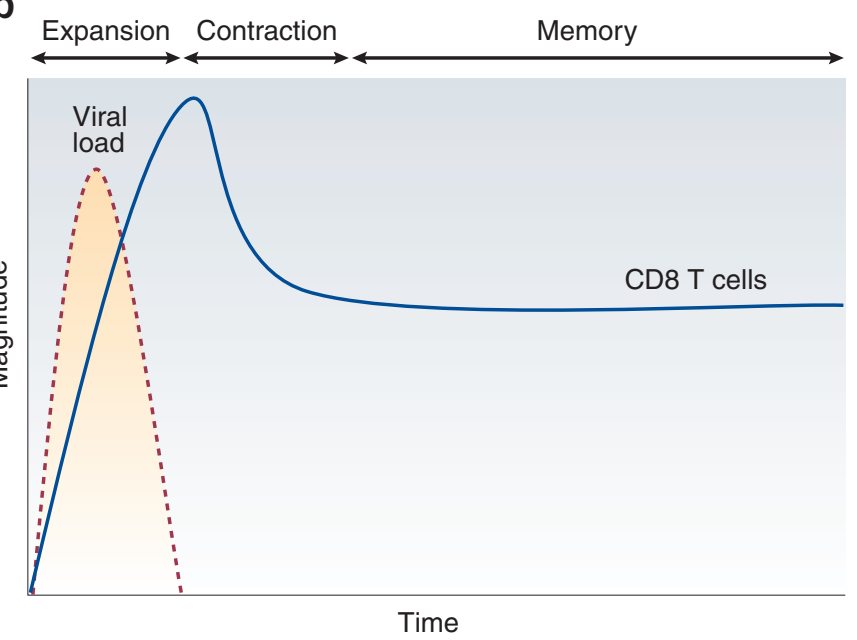

Figure 1 Variability in vaccine elicited responses and phases of CD8 T cell responses. (a) Variability of the peak immune responses in macaques elicited by a DNA-MVA vaccine for the HIV Gag protein compared with that elicited by the DryVax smallpox vaccine. Data for the HIV vaccine are taken from ref. 17 and for the DryVax vaccine from unpublished observations by R. R. Amara and P. Nigam. Macaques were not preselected for histocompatibility type. (b) Three phases of CD8 T cell responses that occur in LCMV-infected mice. The inducing antigen (viral load) is shown in red. Expansion and contraction typically occur in weeks, whereas memory lasts for the lifetime of the mouse. The schematic is adapted from ref. 87 and represents the population of CD8 cells present in the spleen.

\section{Generation of memory cells and phases of $\mathrm{T}$ cell responses}

The goal of $\mathrm{T}$ cell vaccines is to generate long-lived memory CD8 cells capable of recognizing and rapidly expanding to combat an infection ${ }^{26,27}$. In studies in inbred mice, the generation of a memory $\mathrm{T}$ cell response is comprised of three distinct phases: expansion, contraction and differentiation into memory (Fig. 1b). In a primary $\mathrm{T}$ cell response, naive $\mathrm{T}$ cells encounter antigen in lymphoid tissue, where their unique $\mathrm{T}$ cell receptor recognizes its cognate MHC-peptide complex in the context of a 'professional' antigen-presenting cell ${ }^{28,29}$. Signaling through the $\mathrm{T}$ cell receptor as well as costimulatory molecules initiates clonal expansion and differentiation into effector cells that secrete antiviral cytokines such as interferon (IFN)- $\gamma$ and possess ex vivo killing potential. As antigen is cleared, the T cell response contracts (10- to 20 -fold) and differentiates into memory that can last for the lifetime of a mouse (Fig. 1b). Secondary responses have phases like those seen for primary responses, except that expansion can be more rapid and the contraction is (three- to fivefold) less severe ${ }^{30,31}$. Similar phases of primary responses are seen for human and macaque recipients of the smallpox vaccine in whom longitudinal studies show expansion of vaccine-elicited $T$ cells in the first 2 weeks after vaccination, followed by contraction and memory ${ }^{32}$. Cross-sectional studies in humans suggest that the memory response can persist for more than 50 years ${ }^{32-35}$.

Three parameters are important for vaccine-induced memory responses: frequency, phenotype and persistence of memory cells. The frequency of a memory response reflects both the burst size of its effector response and the extent of its contraction into memory. In the lymphocytic choriomeningitis virus (LCMV) mouse model, about one-tenth of the peak effector cells differentiate into long-lived memory cells ${ }^{26}$ (Fig. 1b). The primary determinant of burst size is the amount of antigen present during priming ${ }^{36}$. The amount of required antigen is, in turn, influenced by the extent of costimulation and the cytokine milieu, which can reduce the activation threshold for antigen ${ }^{37,38}$. During the contraction phase, the extent of cell death ${ }^{39}$ is influenced by the interleukin (IL)2 family of cytokines (IL-2, IL-4, IL-7 and IL-15) ${ }^{40-43}$, tumor necrosis factor family molecules (CD27 and CD154) ${ }^{10}$ and effector cell molecules (perforin and IFN- $\gamma)^{44-46}$.

Human memory cells are highly heterogeneous and are still undergoing characterization for lineage relationships. Three categories of memory CD8 cells have been defined based on the expression of CD45RA, a tyrosine phosphatase that has a role in $\mathrm{T}$ cell receptor-mediated signaling, and CCR7, a chemokine receptor that supports trafficking though secondary lymphoid tissue $\mathrm{e}^{47-50}$ (Table 1 ). $\mathrm{T}_{\mathrm{EM}}$, or effector memory cells,

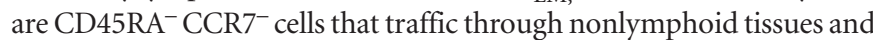
have high ex vivo cytotoxic potential (perforin ${ }^{+}$) but relatively poor proliferative capacity. These are the memory cells that monitor tissues for the presence of their specific pathogen. $\mathrm{T}_{\mathrm{CM}}$, or central memory cells, are CD45RA ${ }^{-} \mathrm{CCR}^{+}$cells that traffic through lymph nodes, possess high proliferative potential and do not possess immediate ex vivo cytotoxic potential (perforin ${ }^{\text {lo }}$ or perforin ${ }^{-}$). These are the memory cells that recognize antigen in lymph nodes and undergo rapid expansion for the generation of large numbers of effector cells. $\mathrm{T}_{\mathrm{RAEM}}$, or CD45RA ${ }^{+}$ effector memory cells (sometimes termed 'terminal memory' cells), are $\mathrm{CD} 4 \mathrm{RA}^{+} \mathrm{CCR}^{-}$cells that traffic through nonlymphoid tissues, possess very rapid ex vivo cytotoxic potential (perforin ${ }^{++}$), but have limited proliferative potential ${ }^{51,52}$ except in the presence of CD4 help, IL-2, IL-15 or IL-21 (ref. 53).

The proportions of memory cells in each category reflect the persistence of antigen. $\mathrm{T}_{\mathrm{CM}}$ predominate in infections in which antigen is cleared, such as in influenza virus infections; $\mathrm{T}_{\mathrm{EM}}$ predominate when relatively high levels of antigen persist, such as in HIV infections; and $\mathrm{T}_{\text {RAEM }}$ are seen when low levels of antigen persist, such as in cytomegalovirus infections ${ }^{49,54}$. In adoptive transfer experiments in mice, $\mathrm{T}_{\mathrm{CM}}$ are more effective at controlling LCMV infections than $\mathrm{T}_{\mathrm{EM}}$, presumably because of their potential to expand and generate large numbers of effector cells ${ }^{55}$. The type of memory cell that will be most effective at controlling other pathogens remains to be determined.

Memory CD4 and CD8 T cells can persist for decades in the absence of obvious re-exposure to antigen ${ }^{32-34}$. This persistence is believed to be achieved by cytokine-dependent homeostatic proliferation mediated by the IL-2 family cytokines IL-7 and IL-15 (refs. 56,57). CD4 $\mathrm{T}$ cells are also needed for the maintenance of a memory response $\mathrm{e}^{8,9}$. And finally, studies in mice suggest that memory responses, especially those trafficking through lymph nodes, are limited to a defined pool size, the detailed constitution of which can be influenced by intercurrent infections ${ }^{27}$. 
Table 1 Categories of human CD8 $\mathrm{T}$ cells and their associated phenotypes

\begin{tabular}{|c|c|c|c|c|c|}
\hline Characteristic & Naive & Effector & Central memory & Effector memory & RA effector memory \\
\hline Marker & ${\mathrm{CD} 45 \mathrm{RA}^{+}, \mathrm{CCR}^{+}}^{+}$ & $\mathrm{CD}^{2} 5 \mathrm{RA}^{-}, \mathrm{CCR}^{-}$ & ${\mathrm{CD} 45 \mathrm{RA}^{-}, \mathrm{CCR}^{+}}^{+}$ & $\mathrm{CD}^{2} 5 \mathrm{RA}^{-}, \mathrm{CCR}^{-}$ & $\mathrm{CD}^{2} 5 \mathrm{RA}^{+}, \mathrm{CCR}^{-}$ \\
\hline \multicolumn{6}{|l|}{ Cytokine production } \\
\hline IFN- $\gamma$ & $+/-($ slow $)$ & ++++ (rapid) & +++ (rapid) & +++ (rapid) & ++ (rapid) \\
\hline IL-2 & +++ (slow) & + (rapid) & +++ (rapid) & + (rapid) & - \\
\hline \multicolumn{6}{|l|}{ Killing potential } \\
\hline Perforin & - & +++ & + & ++ & +++ \\
\hline \multicolumn{6}{|l|}{ Survival potential } \\
\hline $\mathrm{Bcl}-2$ & +++ & Low/- & ++++ & ++ & + \\
\hline \multicolumn{6}{|l|}{ Proliferation potential } \\
\hline Antigen-driven & Rapid & Rapid & Rapid & Slow & Slow \\
\hline Homeostatic & $+/-$ & $+/-$ & ++++ & ++ & $?$ \\
\hline $\begin{array}{l}\text { Trafficking outside of } \\
\text { spleen and blood }\end{array}$ & Lymph node & Tissue & Lymph node & Tissue & Tissue \\
\hline
\end{tabular}

\section{Types of $T$ cell vaccines}

Five types of vaccines have good potential to elicit CD8 T cells: attenuated viruses or bacteria (live attenuated vaccines), replication-competent and replication-defective recombinant viruses or bacteria (live vectored vaccines), DNA vaccines and vaccines that use one agent for priming and a second agent for boosting (heterologous prime-boost vaccines) (Table 2). Each of these has the common feature of producing antigens in cells of the immunized host for presentation on MHC class I antigens. But each has distinctive requirements for dose, boosting and the use of adjuvants, as well as different safety issues and potentials for being limited by pre-existing immunity (Table 2 ). Of these vaccines, only live attenuated vaccines are licensed for human use and these have antibody, not CD8 T cells, as accepted correlates for protection.

In general, safety issues, dose and the need for boosting and adjuvants are related to replication potential. Replicating vaccines are less promised hosts and reverting to virulence. But because they replicate, they typically achieve sufficient antigen and immune stimulation for long-lasting immune responses with single low doses $\left(10^{4}-10^{6}\right.$ infectious units) in the absence of adjuvants. In contrast, nonreplicating live vectored vaccines achieve only one round of infection and typically require high doses $\left(10^{8}-10^{10}\right.$ infectious units) and booster immunizations to achieve sufficient antigen to drive immune responses. DNA vaccines, which act by transfection, also require high doses ( $100 \mu \mathrm{g}-1 \mathrm{mg}$ of DNA or $10^{13}-10^{14}$ molecules) and booster immunizations. These replicationdefective vaccines can be improved by adjuvants, which increase the efficiency of infection or transfection, or provide inflammatory responses for the antigen-presenting cell ${ }^{58}$. But because they do not replicate, they do little harm to their host and have excellent safety records if not used in excessive amounts. Heterologous prime-boost vaccines combine DNA priming with a live vectored boost, or two different live vectors to prime and boost a response $\mathrm{e}^{59,60}$. These vaccines elicit very good $\mathrm{T}$ cell and antibody responses because memory cells established by the first agent are boosted by the second agent with no immunological interference other than to the common vaccine insert.

The two currently most popular recombinant viral vectors, modified vaccinia Ankara (MVA) and an adenovirus 5 vector (Ad5), are both replication defective in humans (see ref. 3 for examples of other viral vectors). The Ad5 vectors are limited to relatively short vaccine inserts $(<5 \mathrm{~kb})$ and may require more than one vector to adequately present foreign sequences, whereas MVA can tolerate larger inserts. Both can become unstable in the presence of inserted foreign sequences and the effectiveness of both can be limited by pre-exisiting host immunity ${ }^{61}$. This is particularly important for the Ad5 vector, for which $45 \%$ of Americans and up to $90 \%$ of people in the developing world have pre-existing immunity. This fact has led to the exploration of the use

Table 2 Vaccines that induce CD8 T cells

\begin{tabular}{|c|c|c|c|c|c|}
\hline Type of vaccine & Dose & $\begin{array}{l}\text { Need for } \\
\text { boost }^{a}\end{array}$ & $\begin{array}{l}\text { Need for } \\
\text { adjuvants }\end{array}$ & Risks & Comment \\
\hline Live-attenuated & Low & No & No & $\begin{array}{l}\text { Reversion to virulence, disease } \\
\text { in immunocompromised }\end{array}$ & $\begin{array}{l}\text { Examples include vaccines for smallpox, yellow fever, oral polio, measles, } \\
\text { mumps, rubella and varicella }\end{array}$ \\
\hline $\begin{array}{l}\text { Replication competent } \\
\text { live-vectored }\end{array}$ & Low & No & No & $\begin{array}{l}\text { Reversion to virulence, disease } \\
\text { in immunocompromised }\end{array}$ & $\begin{array}{l}\text { No examples in human use, yellow fever virus vectors with Japanese } \\
\text { encephalitis virus, dengue virus or West Nile virus are in human trials }{ }^{86}\end{array}$ \\
\hline $\begin{array}{l}\text { Replication-defective } \\
\text { live-vectored }\end{array}$ & High & Varies & No & Low & $\begin{array}{l}\text { Most live-vectored vaccines have problems with pre-existing immunity unless } \\
\text { the parent for the vector has been eradicated and vaccinations stopped (as for } \\
\text { smallpox) or the vector is developed from a virus from another species }\end{array}$ \\
\hline DNA & High & Yes & Helpful & Low & $\begin{array}{l}\text { DNA vaccines, despite their ease of development, have not proven as robust } \\
\text { in humans as rodents. Thus these vaccines need further development or use } \\
\text { in heterologous prime-boost regimens. They do, however, focus the entire } \\
\text { immune response on the vaccine insert and do not have problems with pre- } \\
\text { existing immunity }\end{array}$ \\
\hline $\begin{array}{l}\text { Heterologous prime- } \\
\text { boost }\end{array}$ & High & Yes & Helpful & Varies & $\begin{array}{l}\text { Immense potential to elicit high titer } \mathrm{T} \text { and } \mathrm{B} \text { cell responses. Major limitation } \\
\text { is the need for the use of two different agents in the correct order and interval }\end{array}$ \\
\hline
\end{tabular}




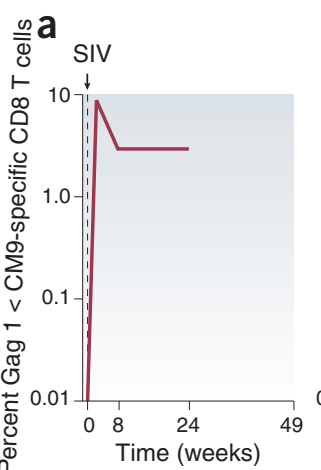

b $\operatorname{SIV} \Delta 3$

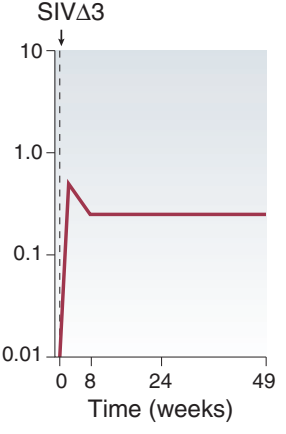

d
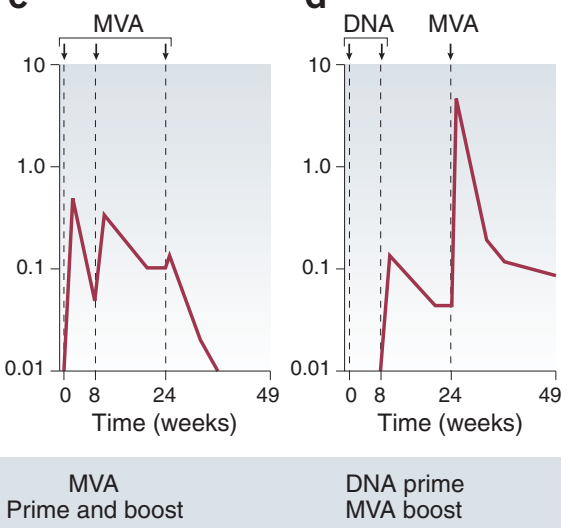

DNA prime

MVA boost

virus model

Relevant target for immune response

Persistence of immunogen

Gag-CM9-specific CD8:

Contraction

CCR7 positive
Memory response focuses boost on vaccine insert

No
Expansion
SIV239

- $\quad$ All regions

Yes

Yes
(High)

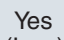

Yes
(Low)

Vaccine insert

No

High

$<10$-fold $<10$-fold

$<5 \%$

$\begin{gathered}\text { Low } \\ <10-\text {-fold } \\ 40 \%\end{gathered}$
4

Low
20-40-fold
NA

Figure 2 Patterns of CD8 T cells found in the blood of $A^{*} 01$ macaques following a SIV infection and the administration of three different SIV or SHIV vaccines. The panels show $T$ cell responses for (a) a live SIV infection, (b) a live, attenuated SIV vaccine, (c) a recombinant MVA vaccine and (d) a DNA-MVA vaccine. The T cell responses are against a common immunodominant epitope (Gag-CM9) and are measured by tetramer staining ${ }^{65}$. All of the studied agents have the same Gag gene. The timing and the number of doses of vaccines are indicated above the panels and by the dotted vertical lines. The virus infection and live-attenuated vaccine are simian immunodeficiency virus 239 (SIV239) and SIV239 3 , respectively 88 . The MVA vaccine is a recombinant with the Gag-Pol and Env genes of SHIV-89.6 and delivered at $2 \times 10^{8}$ infectious units per dose. The DNAMVA vaccine consisted of priming with $2.5 \mathrm{mg}$ of SHIV-89.6 DNA and boosting with $2 \times 10^{8}$ IU of the SHIV-89.6 MVA recombinant. Data are taken from refs. 15,89 and R. Amara, V. Pillai \& H. Robinson, unpublished observations. NA, not available. of less common serotypes of human adenovirus as well as nonhuman primate adenoviruses for the development of new adeno-based vaccine vectors ${ }^{62,63}$. Pre-existing immunity for MVA is largely limited to individuals who were vaccinated before the cessation of vaccinations for smallpox, which occurred in the 1970s. DNA priming can partially overcome the limitations of pre-existing immunity ${ }^{64}$.

Different vaccines elicit different patterns of CD8 T cells

Different infection and immunization regimens elicit strikingly different patterns of CD8 T cells in primates. These patterns, which are tested for in blood, show the expansion, contraction and memory phases defined for LCMV infections in mice (Fig. 1b). But each vaccine has its own relationships between peak effector and memory responses, which probably reflect unique features in the levels of expression and persistence of different vectors, as well as differences in how vectors interface with antigen-presenting cells through pattern-recognition receptors, such as Toll-like receptors, that initiate inflammatory responses ${ }^{58}$. Not unexpectedly, the pattern of $\mathrm{T}$ cell responses elicited by a vaccine insert is usually determined by its vector, and not the pathogen from which the insert was derived.

Examples of different patterns of CD8 responses in macaques are presented in Figures 2 and 3. These responses include ones for a pathogenic simian immunodeficiency virus (SIV239), a live attenuated SIV infection (SIV239 3 3), a replication-defective poxvirus vaccine (MVA), an IL-2-adjuvanted DNA vaccine and two heterologous prime-boost vaccines, DNA/MVA and DNA/Ad5. In each case, monkeys with the $\mathrm{A}^{\star} 01$ histocompatibility type were used for immunizations and responses were followed using tetramer staining for the $\mathrm{A}^{\star} 01$ immunodominant epitope Gag-CM9 (ref. 65). The patterns in Figure 3 include a challenge with a pathogenic chimera of simian and human immunodeficiency viruses (SHIV-89.6P) ${ }^{66}$.

The patterns of CD8 responses show the elicitation of very high burst sizes of CD8 T cells by the SIV239 infection (Fig. 2a) and the two heterologous prime-boost regimens: DNA-MVA (Fig. 2d) and DNA-Ad5 vaccines (Fig. 3a). For each of these, peak tetramer-stain- ing cells represented 5-30\% of total CD8 T cells. About tenfold lower peak burst sizes were elicited by the live attenuated vaccine (Fig. 2b), by priming and boosting with the recombinant MVA vaccine (Fig. $2 \mathrm{c}$ ), and by priming and boosting with the IL-2-adjuvanted DNA vaccine (Fig. 3b).

The different vaccine regimens also had different patterns of contraction into memory. The single doses of SIV239 and its live attenuated vaccine underwent 10 -fold contractions, similar to that observed after LCMV infections in mice (Fig. 1 b and Fig. 2a,b) ${ }^{26}$. The peak responses for DNA-Ad5 and DNA-IL-2-immunoglobulin underwent smaller contractions, less than fivefold, into memory (Fig. 3a,b). The contraction of the DNA-IL-2-immunoglobulin response was less than typically seen for DNA immunizations (Fig. 3a,c) and consistent with the addition of IL-2 as an adjuvant to limit apoptotic death during contraction ${ }^{67}$. By far the largest contractions were seen for the DNA-MVA and MVA responses, which underwent 20-40-fold reductions (Fig. 2c,d), a phenomenon that also occurs in DNA-poxvirus regimens in which NYVAC (an attenuated vaccinia virus) and ALVAC (a canarypox virus) are used for boosting ${ }^{68}$.

Relatively limited data are available on the phenotypes of the CD8 cells that emerged in the memory cell population. The very high levels of persisting CD8 cells elicited by the pathogenic SIV239 infection showed an 'exhausted' phenotype, as evidenced by their inability to coproduce IFN- $\gamma$ and IL-2 (V. Pillai \& R.R. Amara, unpublished data $)^{21,69,70}$. For the live attenuated SIV infection, the much lower frequency of persisting memory cells were both $\mathrm{T}_{\mathrm{EM}}$ and $\mathrm{T}_{\mathrm{CM}}$ cells. The memory cells for the DNA-MVA immunizations fell predominantly into the $\mathrm{T}_{\mathrm{CM}}$ category. Thus, the three situations for which partial phenotypes are available show three different scenarios for memory responses in the peripheral blood. The chronic SIV infection resulted in a high frequency of exhausted effector memory cells, the chronic live attenuated vaccine in a low frequency of primarily tissue-trafficking effector memory cells and the DNA-MVA vaccine in a low frequency of primarily lymph node- and spleen-trafficking central memory cells. 
b DNA-IL-2-Ig

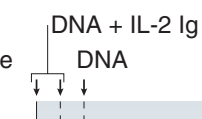

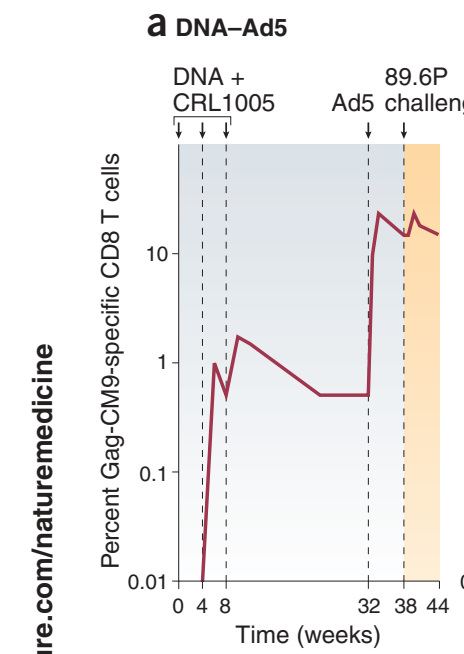

C DNA-MVA

Vaccine

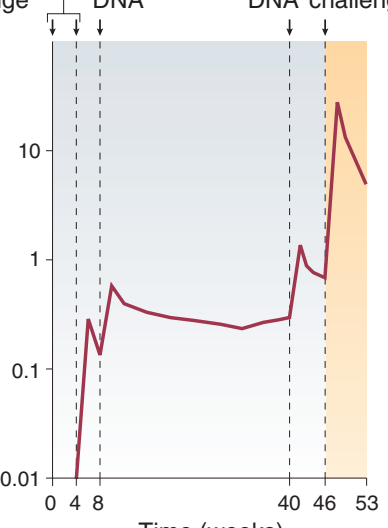

Time (weeks)

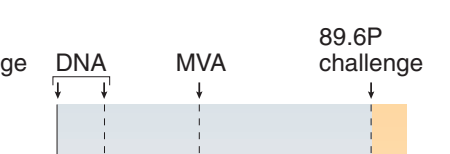

Figure 3 Patterns of CD8 T cells found in the blood of $A^{*} 01$ macaques, both before and after challenge, for three different types of vaccines. The panels show $T$ cell responses for (a) priming with DNA and boosting with a recombinant Ad5 vaccine, (b) priming with an IL-2-immunoglobulin-adjuvanted DNA and boosting with DNA and (c) priming with DNA and boosting with a recombinant MVA vaccine. Challenge was with SHIV-89. $6 \mathrm{P}^{66}$. The postchallenge period of the trial is highlighted in orange. Panels show T cell responses against the same immunodominant epitope (Gag-CM-9) used to follow T cell responses in Figure 2. The DNA-Adeno vaccine consisted of priming with $5 \mathrm{mg}$ of SIV gag DNA adjuvanted with CRL1005 and boosting with $1 \times 10^{11}$ particles of rAd5-gag ${ }^{90}$. The DNA-IL-2-immunoglobulin (Ig) vaccine used IL-2-immunoglobulin-adjuvanted DNA priming $(5 \mathrm{mg}$ each of SIV gag DNA, HIV 89.6P env DNA and IL-2-immunoglobulin fusion protein DNA) and DNA boosting (5 mg each of SIV gag DNA, HIV 89.6P env DNA $)^{91,92}$. The DNA-MVA vaccine used $2.5 \mathrm{mg}$ of SHIV-89.6 DNA (gag, pol, env, tat, rev, vif, vpr and $v p u$ )for priming and $2 \times 10^{8}$ plaque-forming units of MVA/SHIV-89.6 (gag, $\mathrm{pol}$ and env)for boosting ${ }^{89}$. Challenges for the DNA-Adeno and DNA-IL-2-immunoglobulin were by intravenous inoculation of $\sim 100$ infectious unit of SHIV-89.6P90,92 at 6 weeks after the last inoculation. Challenge for the DNA-MVA immunizations was by intrarectal inoculation of $\sim 20$ intrarectal doses of SHIV-89.6P89 at 7 months after the last inoculation. Data are adapted from refs. $89,90,92$. NT, not tested.

\section{No simple correlate for protection}

Most of the experience with the protective potential of $\mathrm{T}$ cell vaccines in nonhuman primates is for immunodeficiency virus challenges, where remarkably different patterns of $\mathrm{T}$ cell responses have rallied to control challenges with SHIV-89.6P. In the examples in Figure 3, the frequencies of memory CD8 cells in peripheral blood on the day of challenge differed by about 100 -fold for the three vaccine regimens. For the DNA-Ad5 regimen, tetramer-staining cells were $14 \%$ of the total CD8 T cells (Fig. 3a); for the DNA-IL-2-immunoglobulin regimen, a much lower $0.6 \%$ of total CD8 T cells (Fig. 3 b) and for the DNA-MVA regimen, a mere $0.2 \%$ of total CD8 T cells (Fig. 3c). At 1 week after challenge, the virus-specific $\mathrm{CD} 8 \mathrm{~T}$ cells in the DNA-MVA vaccinated macaques had virtually disappeared from the blood (Fig. 3c). But by 2 weeks after challenge, each of the vaccinated groups had similar high levels of responding Gag-CM9 tetramer-staining cells ( $\sim 20 \%$ of total CD8 T cells), and by 6 weeks after challenge, these were again contracting as viral loads were contained (Fig. $3 \mathrm{a}-\mathrm{c})$. This contraction was more pronounced for the DNA-MVA and DNA-IL-2-immunoglobulin-adjuvanted groups than the DNA-Ad5 group (Fig. 3a-c). In the DNA-MVA group, the temporary disappearance from the blood followed by the exceptionally rapid expansion of the responding cells suggested that these cells (primarily $\mathrm{T}_{\mathrm{CM}}$ cells) had left the blood for lymph nodes where they expanded before reappearing. The very low postvaccine and challenge contraction for the DNA-Ad5-elicited response suggests the continuing presence of antigen (either persisting Ad5 vector or persisting challenge virus) and/or an antiapoptotic environment limiting the contraction of the $\mathrm{CD} 8$ response.
Thus, very different frequencies of CD8 responses before challenge resulted in similar frequencies after challenge and showed no clear correlate for control of the SHIV-89.6P challenge. The absence of a simple CD8 correlate, such as the frequency of tetramer-staining cells in peripheral blood, does not belie the importance of $\mathrm{CD} 8$ responses to the observed control. The DNA-IL-2-immunoglobulin-immunized macaques lost control when virus underwent CD8 escape ${ }^{71}$. In the DNA-MVA-vaccinated macaques, virus rapidly re-emerged when CD8 T cells were experimentally depleted (R.R. Amara \& H.L. Robinson, unpublished observations). For DNA-Ad5 vaccines, control of SHIV-89.6P is histocompatibility-type dependent and more effective in $\mathrm{A}^{\star} 01$ macaques than in macaques that do not recognize the immunodominant Gag-CM9 epitope $^{72}$.

Assays for $\mathrm{T}$ cell vaccines. Testing for $\mathrm{CD} 8 \mathrm{~T}$ cell responses is much more challenging than testing for antibody responses. Recently developed ex vivo assays directly test for the frequency and phenotype of responding T cells (Fig. 4), whereas restimulation assays, which involve 5 or more days of cell culture, are used to test for proliferative potential and killing capacity (Fig. 4). Stimulations are typically conducted by infecting peripheral blood mononuclear cells (PBMCs) with the target agent or a recombinant virus expressing a vaccine insert or by culturing PBMCs with pools of overlapping peptides (typically 15-mers overlapping by 11 so as not to miss any CD8 or CD4 epitopes). In some instances, B cell lines are immortalized for each participant and then used to both process and present antigens and to serve as targets for cell killing. Assays are best conducted on fresh PBMCs. They can be conducted on frozen 


\section{REVIEW}

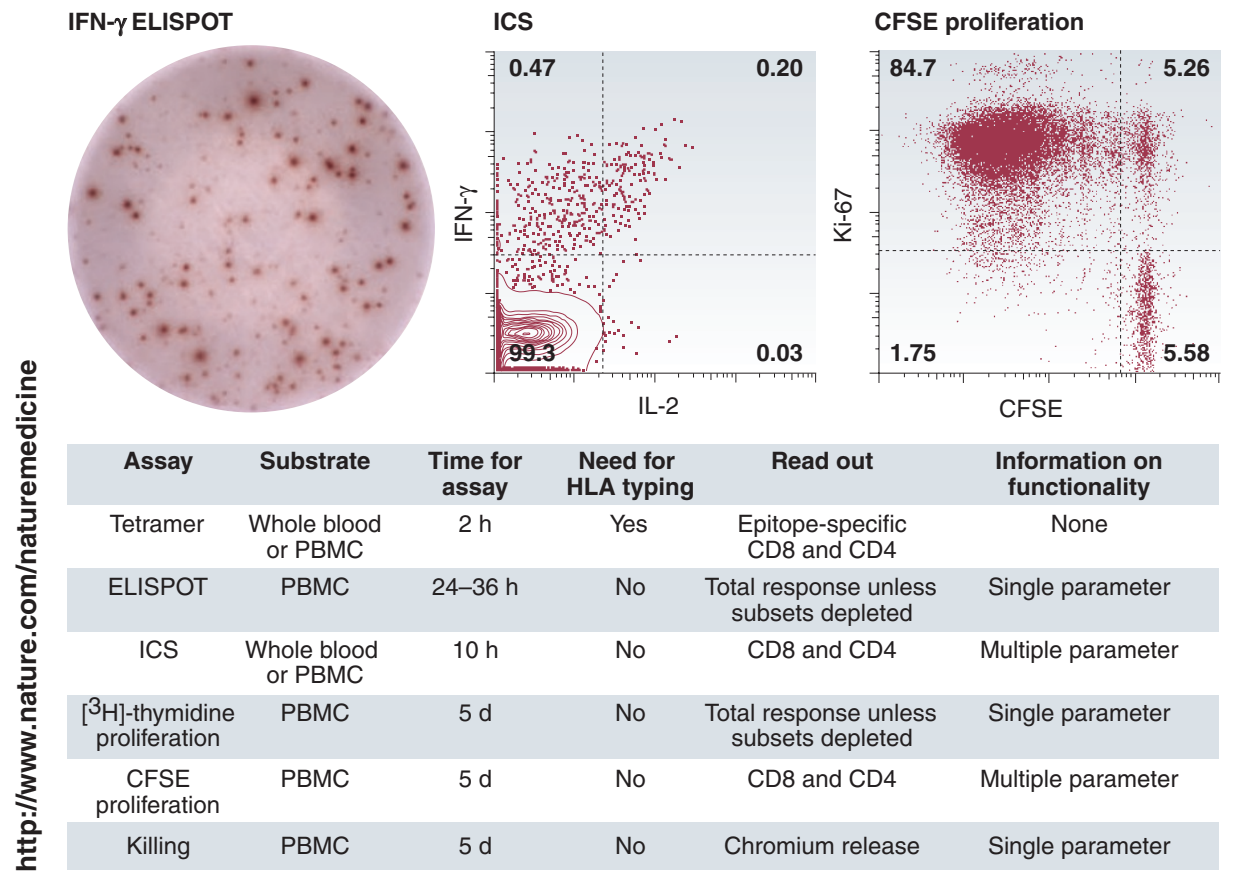

Figure 4 Assays for $T$ cell responses. Examples of an IFN- $\gamma$ ELISPOT assay, an ICS and a CFSE proliferation assay are shown. All assays test for responses to peptide stimulations. For the ELISPOT assay, the spots represent IFN- $\gamma$, which has been secreted by an individual cell in response to stimulation. For the ICS assay, each dot represents a CD8 T cell and the position in the FACScan represents the cytokines produced in response to peptide stimulation. Cells in the upper left quadrant are IFN- $\boldsymbol{\gamma}$-producing cells, cells in the upper right quadrant are IFN- $\gamma$ - and IL-2-producing cells and cells in the lower right quadrant are cells that produce only IL-2 (this cell type is rare among CD8 cells). The strength of cytokine production by individual cells is reflected by the positions of cells in their respective quadrants. For the CFSE assay, cells were prestained with CFSE and then stimulated with peptide for 6 days. Following the stimulation, CD8 cells were analyzed for CFSE and staining with $\mathrm{Ki}-67$ (a marker for dividing cells). Cells with the least intense staining for CFSE (furthest to the left in the FACScan) and positive for Ki-67 (upper quadrants) are the ones that have undergone the most division. $h$, hours; d, days. samples, however, with the caveat that cells revived from the peak effector phase of a response perform less well than cells revived from the memory phase of a response.

The various assays offer different information. The tetramer assay directly tests samples for the frequency of CD8 T cells with a specific $\mathrm{T}$ cell receptor ${ }^{73}$ and, if combined with multicolor flow cytometry, offers the opportunity to determine the phenotypes of cells without in vitro stimulation. But tetramer staining is limited by the availability of reagents and the need to tailor tests to the histocompatibility type of each trial participant. The ELISPOT and intracellular cytokine assays use short term in vitro stimulations to assay the frequency and cytokine profiles of responding cells. The ELISPOT assay scores spots of captured cytokine produced by individual cells whereas intracellular cytokine staining (abbreviated as ICS or ICC) uses flow cytometry to profile individual cells for surface markers and the production of cytokines $^{74,75}$. Among the restimulation assays, the carboxyl fluorescein diacetate succinimidyl (CFSE) proliferation assay, a flow cytometry assay, has the ability to not only monitor proliferation (by dilution of the CFSE stain) but to determine the type of the proliferating cell ${ }^{76}$.

Of the above assays, the IFN- $\gamma$ ELISPOT is the workhorse that has been validated for use in vaccine trials. But 'ancillary' multicolor ICS assays are proving invaluable for characterizing the functions of responding cells $\mathrm{s}^{77}$. The easiest time to assay responses is at the peak effector response, when responses are highest and volunteers are in recent communication with trial coordinators (Figs. 2 and 3). Although this peak response does not represent the memory response, it does have a defined relationship (albeit different for different vectors) with the memory response (Figs. 1-3).

\section{Early studies in humans}

The results of early clinical trials of T cell vaccines for HIV, malaria and tuberculosis are being reported at meetings, and beginning to be published. DNA vaccines, which have the largest number of reports, have proved to be safe ${ }^{78,79}$, but only weakly immunogenic, even when used at milligram doses ${ }^{80-82}$. MVA vaccines have proved safe, but weakly immunogenic ${ }^{80-82}$. Ad5 vaccines have elicited responses, but shown reduced immunogenicity in the presence of pre-existing immunity. By far the most promise has been for heterologous prime-boost combinations. These DNA-MVA and fowlpox virus (FP9)-MVA malaria vaccines have elicited peak responses that are 50 -fold higher ( 200 IFN- $\gamma$ ELISPOTS per million PBMC) than baseline in endemic populations ${ }^{83}$. These responses are higher than seen for the DNA-MVA vaccine in a malaria-naive British population $^{82}$. The malaria studies used a fusion of a malaria protein and a synthetic string of epitopes (tailored to the histocompatibility type of the target population) to express antigens and found the natural protein much more effective than the string of epitopes at eliciting T cells ${ }^{82,84}$.

By far the most effective of the heterologous prime-boost vaccine strategies used a tuberculosis model, in which priming was with the tuberculosis vaccine Bacille Calmette-Guérin (BCG) and boosting with MVA expressing the 85A antigen of Mycobacterium tuberculosis ${ }^{85}$. This combination elicited a high-titer CD4 IFN- $\gamma$ ELISPOT response. Interestingly, the burst of peak effector cells did not show as profound a contraction as observed for CD8 cells elicited by MVA boosts in macaques (Fig. 2). This could reflect the persistence of the BCG prime, the response being predominantly CD4 or other factors. But the power of the response emphasizes the promise of heterologous prime-boost regimens for $\mathrm{T}$ cell vaccines for humans.

\section{Future directions}

The evaluation of the ability of $\mathrm{T}$ cells to control infections is still in its infancy. A major limitation for this new approach is the complexity of the assays used to score responding $\mathrm{T}$ cells and the failure of these assays, as currently used, to provide a single correlate for protection. Also, many diseases for which $\mathrm{T}$ cell vaccines are needed (such as HIV and tuberculosis) are diseases that do not allow experimental human challenge, necessitating vaccine development using animal models that may not accurately represent human infections. This means that the ultimate determination of efficacy will need to come from large-scale human trials. Nevertheless, remarkable progress is being made in our ability to elicit and score antimicrobial $\mathrm{T}$ cells with true hope for the realization of vaccines for such important causes of human morbidity and mortality as HIV, tuberculosis and malaria. 


\section{ACKNOWLEDGMENTS}

We are indebted to R. Ahmed, J. Altman, F. Villinger, V. Pillai, L. Lai and J. Zhao for critical review of the manuscript and to H. Drake-Perrow for administrative assistance. This work was supported by National Public Health Service Integrated Preclinical/Clinical AIDS Vaccine Development Grants P01-AI 43045 and P01AI49364, the Emory/Atlanta Center for AIDS Research, P30 DA 12121, and the Yerkes National Primate Research Center base grant, P51 RR000165.

\section{COMPETING INTERESTS STATMENT}

The authors declare competing financial interests (see the Nature Medicine website for details).

Published online at http://www.nature.com/naturemedicine/

1. Nossal, G.J. The Global Alliance for Vaccines and Immunization-a millennial challenge. Nat. Immunol. 1, 5-8 (2000).

2. Burton, D.R. et al. HIV vaccine design and the neutralizing antibody problem. Nat. Immunol. 5, 233-236 (2004).

3. Robinson, H.L. New Hope for an AIDS Vaccine. Nat. Rev. Immunol. 2, 239-250 (2002)

4. Letvin, N. L. Progress toward an HIV vaccine. Annu. Rev. Med. 56, 213-223 (2005).

5. Bourgeois, C., Rocha, B. \& Tanchot, C. A role for CD40 expression on CD8+ T cells in the generation of $C D 8^{+} T$ cell memory. Science 297, 2060-2063 (2002).

6. Janssen, E.M. et al. CD4+ T cells are required for secondary expansion and memory in CD8+ T lymphocytes. Nature 421, 852-856 (2003).

7. Shedlock, D.J. \& Shen, H. Requirement for CD4 T cell help in generating functional CD8 T cell memory. Science 300, 337-339 (2003).

8. Sun, J.C. \& Bevan, M.J. Defective CD8 T cell memory following acute infection without CD4 T cell help. Science 300, 339-342 (2003).

9. Sun, J.C., Williams, M.A. \& Bevan, M.J. CD4 ${ }^{+}$T cells are required for the maintenance, not programming, of memory CD8+ T cells after acute infection. Nat. Immunol. 5, 927-933 (2004)

10. Borrow, P. et al. CD4OL-deficient mice show deficits in antiviral immunity and have an impaired memory CD8+ CTL response. J. Exp. Med. 183, 2129-2142 (1996).

11. Rubbert, A. et al. Multifactorial nature of noncytolytic $C D 8^{+} T$ cell-mediated suppression of HIV replication: beta-chemokine-dependent and -independent effects. AIDS Res. Hum. Retroviruses 13, 63-69 (1997).

12. Levy, J.A., Mackewicz, C.E. \& Barker, E. Controlling HIV pathogenesis: the role of the noncytotoxic anti-HIV response of CD8+ T cells. Immunol. Today 17, 217-224 (1996).

13. Pal, R. et al. Inhibition of HIV-1 infection by the beta-chemokine MDC. Science $\mathbf{2 7 8}$, 695-698 (1997).

14. Amara, R.R. et al. Critical role for Env as well as Gag-Pol in control of a simian-human immunodeficiency virus $89.6 \mathrm{P}$ challenge by a DNA prime/recombinant modified vaccinia virus Ankara vaccine. J. Virol. 76, 6138-6146 (2002).

15. Amara, R.R. et al. Different patterns of immune responses but similar control of a mucosal immunodeficiency virus challenge by MVA and DNA/MVA vaccines. J. Virol. 76, 7625-7631 (2002)

16. Smith, J.M. et al. Multiprotein HIV-1 Clade B DNA/MVA Vaccine: Construction, Safety and Immunogenicity. AIDS Res. Hum. Retroviruses 20, 654-665 (2004).

17. Smith, J.M. et al. DNA/MVA vaccine for HIV type 1: effects of codon-optimization and the expression of aggregates or virus-like particles on the immunogenicity of the DNA prime. AIDS Res. Hum. Retroviruses 20, 1335-1347 (2004).

18. Sette, A. \& Fikes, J. Epitope-based vaccines: an update on epitope identification, vaccine design and delivery. Curr. Opin. Immunol. 15, 461-470 (2003).

19. Goulder, P.J. \& Watkins, D.I. HIV and SIV CTL escape: implications for vaccine design. Nat. Rev. Immunol. 4, 630-640 (2004).

20. Lechner, F. et al. Analysis of successful immune responses in persons infected with hepatitis C virus. J. Exp. Med. 191, 1499-1512 (2000).

21. Wherry, E.J., Blattman, J.N., Murali-Krishna, K., van der Most, R. \& Ahmed, R. Viral persistence alters CD8 T-cell immunodominance and tissue distribution and results in distinct stages of functional impairment. J. Virol. 77, 4911-4927 (2003).

22. Kostense, S. et al. Persistent numbers of tetramer+ CD8(+) T cells, but loss of interferon-gamma+ HIV-specific T cells during progression to AIDS. Blood 99, 2505-2511 (2002).

23. Sadagopal, S. et al. Signature for Long-term vaccine-mediated controlof as SHIV-98.6P challenge: Stable low breadth and low frequency T cell response capable of co-producing IFN-gamma and IL-2. J. Virol. 79, 3243-3253 (2005).

24. McKay, P.F. et al. Vaccine protection against functional CTL abnormalities in simian human immunodeficiency virus-infected rhesus monkeys. J. Immunol. 168, 332-337 (2002).

25. Migueles, S.A. et al. HIV-specific CD8+ T cell proliferation is coupled to perforin expression and is maintained in nonprogressors. Nat. Immunol. 3, 1061-1068 (2002).

26. Kaech, S.M., Wherry, E.J. \& Ahmed, R. Effector and memory T-cell differentiation: implications for vaccine development. Nat. Rev. Immunol. 2, 251-262 (2002).

27. Welsh, R.M., Selin, L.K. \& Szomolanyi-Tsuda, E. Immunological memory to viral infections. Annu. Rev. Immunol. 22, 711-743 (2004).

28. Ahmed, R. \& Gray, D. Immunological memory and protective immunity: understanding their relation. Science 272, 54-60 (1996).

29. Banchereau, J. \& Steinman, R.M. Dendritic cells and the control of immunity. Nature 392, 245-252 (1998).

30. Grayson, J.M., Harrington, L.E., Lanier, J.G., Wherry, E.J. \& Ahmed, R. Differential sensitivity of naive and memory CD8 ${ }^{+} \mathrm{T}$ cells to apoptosis in vivo. J. Immunol. 169 , 3760-3770 (2002)

31. Badovinac, V.P., Messingham, K.A., Hamilton, S.E. \& Harty, J.T. Regulation of CD8 T cells undergoing primary and secondary responses to infection in the same host. J. Immunol. 170, 4933-4942 (2003).

32. Amara, R.R., Nigam, P., Sharma, S., Liu, J. \& Bostik, V. Long-lived poxvirus immunity, robust CD4 help, and better persistence of CD4 than CD8 T cells. J. Virol. 78 3811-3816 (2004).

33. Hammarlund, E. et al. Duration of antiviral immunity after smallpox vaccination. Nat. Med. 9, 1131-1137 (2003)

34. Crotty, S. et al. Cutting edge: long-term B cell memory in humans after smallpox vaccination. J. Immunol. 171, 4969-4973 (2003).

35. Demkowicz, W.E., Jr., Littaua, R.A., Wang, J. \& Ennis, F.A. Human cytotoxic T-cell memory: long-lived responses to vaccinia virus. J. Virol. 70, 2627-2631 (1996)

36. Wherry, E.J., Puorro, K.A., Porgador, A. \& Eisenlohr, L.C. The induction of virus-specific $C T L$ as a function of increasing epitope expression: responses rise steadily until excessively high levels of epitope are attained. J. Immunol. 163, 3735-3745 (1999).

37. Pardigon, N. et al. Role of co-stimulation in $\mathrm{CD}^{+} \mathrm{T}$ cell activation. Int. Immunol. 10 , 619-630 (1998).

38. Whitmire, J.K. \& Ahmed, R. Costimulation in antiviral immunity: differential requirements for CD4(+) and CD8(+) T cell responses. Curr. Opin. Immunol. 12, 448-455 (2000).

39. Marrack, P. \& Kappler, J. Control of T cell viability. Annu. Rev. Immunol. 22, 765-787 (2004).

40. Blattman, J.N. et al. Therapeutic use of IL-2 to enhance antiviral T-cell responses in vivo. Nat. Med. 9, 540-547 (2003)

41. Yajima, T. et al. Overexpression of IL-15 in vivo increases antigen-driven memory CD8+ T cells following a microbe exposure. J. Immunol. 168, 1198-1203 (2002).

42. Khan, I.A. \& Casciotti, L. IL-15 prolongs the duration of CD8+ T cell-mediated immunity in mice infected with a vaccine strain of Toxoplasma gondii. J. Immunol. 163, 4503-4509 (1999)

43. Sprent, J., Zhang, X., Sun, S. \& Tough, D. T-cell proliferation in vivo and the role of cytokines. Philos. Trans. R. Soc. Lond. B Biol. Sci. 355, 317-322 (2000).

44. Badovinac, V.P., Tvinnereim, A.R. \& Harty, J.T. Regulation of antigen-specific CD8 ${ }^{+}$ T cell homeostasis by perforin and interferon-gamma. Science 290, 1354-1358 (2000).

45. Kagi, D., Odermatt, B. \& Mak, T.W. Homeostatic regulation of CD8+ T cells by perforin. Eur. J. Immunol. 29, 3262-3272 (1999).

46. Matloubian, M. et al. A role for perforin in downregulating T-cell responses during chronic viral infection. J Virol 73, 2527-36 (1999).

47. Sallusto, F., Lenig, D., Forster, R., Lipp, M. \& Lanzavecchia, A. Two subsets of memory T lymphocytes with distinct homing potentials and effector functions. Nature 401 , 708-712 (1999).

48. Sallusto, F., Geginat, J. \& Lanzavecchia, A. Central memory and effector memory T cell subsets: function, generation, and maintenance. Annu. Rev. Immunol. 22, 745-763 (2004).

49. Appay, V. et al. Memory CD8 ${ }^{+} \mathrm{T}$ cells vary in differentiation phenotype in different persistent virus infections. Nat. Med. 8, 379-385 (2002)

50. Ravkov, E.V., Myrick, C.M. \& Altman, J.D. Immediate early effector functions of virusspecific $\mathrm{CD}^{+}{ }^{+} \mathrm{CR} 7^{+}$memory cells in humans defined by $\mathrm{HLA}$ and $\mathrm{CC}$ chemokine ligand 19 tetramers. J. Immunol. 170, 2461-2468 (2003).

51. Geginat, J., Lanzavecchia, A. \& Sallusto, F. Proliferation and differentiation potential of human $\mathrm{CD} 8^{+}$memory T-cell subsets in response to antigen or homeostatic cytokines. Blood 101, 4260-4266 (2003).

52. Faint, J.M. et al. Memory T cells constitute a subset of the human $C D 8^{+} C D 45 R A^{+}$pool with distinct phenotypic and migratory characteristics. J. Immunol. 167, 212-220 (2001).

53. van Leeuwen, E.M. et al. Proliferation requirements of cytomegalovirus-specific, effector-type human CD8+ T cells. J. Immunol. 169, 5838-5843 (2002).

54. Champagne, P. et al. Skewed maturation of memory HIV-specific CD8 T lymphocytes. Nature 410, 106-111 (2001).

55. Wherry, E.J. et al. Lineage relationship and protective immunity of memory CD8 T cell subsets. Nat. Immunol. 4, 225-234 (2003).

56. Prlic, M., Lefrancois, L. \& Jameson, S.C. Multiple choices: regulation of memory CD8 T cell generation and homeostasis by interleukin (IL)-7 and IL-15. J. Exp. Med. 195 , F49-F52 (2002).

57. Tan, J.T. et al. Interleukin (IL)-15 and IL-7 jointly regulate homeostatic proliferation of memory phenotype $\mathrm{CD} 8^{+}$cells but are not required for memory phenotype $\mathrm{CD} 4^{+}$cells. J. Exp. Med. 195, 1523-1532 (2002).

58. Pulendran, B. Modulating vaccine responses with dendritic cells and Toll-like receptors. Immunol. Rev. 199, 227-250 (2004).

59. Schneider, J. et al. Induction of $\mathrm{CD} 8^{+} \mathrm{T}$ cells using heterologous prime-boost immunisation strategies. Immunol. Rev. 170, 29-38 (1999).

60. Robinson, H.L., Smith, J.M. \& Amara, R.R. AIDS Vaccines: Heterologous Prime/Boost Strategies for raising Protective T Cell Responses. AIDS Rev. 2, 105-110 (2000).

61. Sumida, S.M. et al. Neutralizing antibodies and $C D 8^{+} T$ lymphocytes both contribute to immunity to adenovirus serotype 5 vaccine vectors. J. Virol. 78, 2666-2673 (2004).

62. Shiver, J.W. \& Emini, E.A. Recent advances in the development of HIV-1 vaccines using replication-incompetent adenovirus vectors. Annu. Rev. Med. 55, 355-372 (2004).

63. Reyes-Sandoval, A. et al. Human immunodeficiency virus type 1-specific immune responses in primates upon sequential immunization with adenoviral vaccine carriers of human and simian serotypes. J. Virol. 78, 7392-7399 (2004).

64. Yang, Z.Y. et al. Overcoming immunity to a viral vaccine by DNA priming before vector boosting. J. Virol. 77, 799-803 (2003). 


\section{REVIEW}

65. Allen, T.M. et al. Induction of AIDS virus-specific CTL activity in fresh, unstimulated peripheral blood lymphocytes from rhesus macaques vaccinated with a DNA Prime/ Modified vaccinia virus ankara boost regimen. J. Immunol. 164, 4968-4978 (2000).

66. Reimann, K.A. et al. A chimeric simian/human immunodeficiency virus expressing a primary patient human immunodeficiency virus type 1 isolate env causes an AIDS-like disease after in vivo passage in rhesus monkeys. J. Virol. 70, 6922-6928 (1996).

67. Barouch, D.H. et al. Augmentation and suppression of immune responses to an HIV-1 DNA vaccine by plasmid cytokine/lg administration. J. Immunol. 161, 1875-1882 (1998).

68. Santra, S. et al. Recombinant poxvirus boosting of DNA-primed rhesus monkeys augments peak but not memory T lymphocyte responses. Proc. Natl. Acad. Sci. USA 101, 11088-11093 (2004)

69. Appay, V. et al. HIV-specific CD8(+) T cells produce antiviral cytokines but are impaired in cytolytic function. J. Exp. Med. 192, 63-75 (2000).

. Gruener, N.H. et al. Sustained dysfunction of antiviral CD8 ${ }^{+}$T lymphocytes after infection with hepatitis C virus. J. Virol. 75, 5550-5558 (2001).

71. Barouch, D.H. et al. Eventual AIDS vaccine failure in a rhesus monkey by viral escape from cytotoxic T lymphocytes. Nature 415, 335-339 (2002).

72. Kahn, P. AIDS Vaccines, From Monkeys to People: An Interview with John Shriver. in IAVI Report Vol. 7 10-12 (International AIDS Vaccine Initiative, New York, 2003)

73. Altman, J.D. et al. Phenotypic analysis of antigen-specific T lymphocytes. Science $\mathbf{2 7 4}$ 94-96 (1996).

74. Waldrop, S.L., Pitcher, C.J., Peterson, D.M., Maino, V.C. \& Picker, L.J. Determination of antigen-specific memory/effector $\mathrm{CD} 4^{+} \mathrm{T}$ cell frequencies by flow cytometry: evidence for a novel, antigen-specific homeostatic mechanism in HIV-associated immunodeficiency. J. Clin. Invest. 99, 1739-1750 (1997).

75. Waldrop, S.L., Davis, K.A., Maino, V.C. \& Picker, L.J. Normal human CD4+ memory $\mathrm{T}$ cells display broad heterogeneity in their activation threshold for cytokine synthesis. J. Immunol. 161, 5284-5295 (1998).

76. Lyons, A.B. \& Parish, C.R. Determination of lymphocyte division by flow cytometry. J. Immunol. Methods 171, 131-137 (1994).

77. De Rosa, S.C. et al. Vaccination in humans generates broad T cell cytokine responses. J. Immunol. 173, 5372-5380 (2004).

은 78. MacGregor, R.R. et al. First human trial of a facilitated DNA plasmid vaccine for HIV-1: safety and host response. in Int. Conf. AIDS 11, 23 (abstract no. We.B.293) (1996).
79. MacGregor, R.R. et al. First human trial of a DNA-based vaccine for treatment of human immunodeficiency virus type 1 infection: safety and host response. J. Infect. Dis. 178, 92-100 (1998).

80. Moorthy, V.S. et al. Safety of DNA and modified vaccinia virus Ankara vaccines against liver-stage P. falciparum malaria in non-immune volunteers. Vaccine 21, 1995-2002 (2003).

81. Moorthy, V.S. et al. Safety and immunogenicity of DNA/modified vaccinia virus ankara malaria vaccination in African adults. J. Infect. Dis. 188, 1239-1244 (2003).

82. McConkey, S.J. et al. Enhanced T-cell immunogenicity of plasmid DNA vaccines boosted by recombinant modified vaccine virus Ankara in humans. Nat. Med. 9, 729-735 (2003).

83. Moorthy, V.S. et al. Phase 1 evaluation of 3 highly immunogenic prime-boost regimens, including a 12-month reboosting vaccination, for malaria vaccination in Gambian men. J. Infect. Dis. 189, 2213-2219 (2004).

84. Moore, A.C. \& Hill, A.V. Progress in DNA-based heterologous prime-boost immunization strategies for malaria. Immunol. Rev. 199, 126-143 (2004).

85. McShane, H. et al. Recombinant modified vaccinia virus Ankara expressing antigen 85A boosts BCG-primed and naturally acquired antimycobacterial immunity in humans. Nat. Med. 10, 1240-1244 (2004).

86. Lai, C.J. \& Monath, T.P. Chimeric flaviviruses: novel vaccines against dengue fever, tick borne encephalitis, and Japanese encephalitis. Adv. Virus Res. 61, 469-509 (2003).

87. Kaech, S.M. \& Ahmed, R. Memory CD8+ T cell differentiation: initial antigen encounter triggers a developmental program in naive cells. Nat. Immunol. 2, 415-422 (2001).

88. Wyand, M.S., Manson, K.H., Garcia-Moll, M., Montefiori, D. \& Desrosiers, R.C. Vaccine protection by a triple deletion mutant of simian immunodeficiency virus. J. Virol. 70, 3724-3733 (1996).

89. Amara, R.R. et al. Control of a Mucosal Challenge and Prevention of AIDS by a Multiprotein DNA/MVA Vaccine. Science 292, 69-74 (2001).

90. Shiver, J.W. et al. Replication-incompetent adenoviral vaccine vector elicits effective anti-immunodeficiency virus immunity. Nature 415, 331-335 (2002).

91. Barouch, D.H. et al. Augmentation of immune responses to HIV-1 and simian immunodeficiency virus DNA vaccines by IL-2/lg plasmid administration in rhesus monkeys. Proc. Natl. Acad. Sci. USA 97, 4192-4197 (2000).

92. Barouch, D.H. et al. Control of viremia and prevention of clinical AIDS in rhesus monkeys by cytokine-augmented DNA vaccination. Science 290, 486-492 (2000). 